\title{
CEPAS ATENUADAS DE Babesia bovis E Babesia bigemina E DE Anaplasma centrale COMO IMUNÓGENOS NO CONTROLE DA TRISTEZA PARASITÁRIA BOVINA
}

\section{ATTENUATED STRAINS OF Babesia bovis AND Babesia bigemina AND OF Anaplasma centrale AS IMMUNOGENS FOR TICK BORNE DISEASE CONTROL}

\author{
Ana Maria Sastre Sacco ${ }^{1}$ Raul Henrique Kessler ${ }^{2}$ Cláudio Roberto Madruga ${ }^{2}$
}

RESUMO

Neste trabalho foram avaliadas cepas atenuadas de Babesia bovis e B. bigemina e Anaplasma centrale como imunógenos a serem utilizados no controle da Tristeza Parasitária Bovina. O processo de imunização demonstrou ser inócuo, imunogênico e eficiente, pelo menos no que diz respeito às babesias, pois protegeu os animais vacinados frente ao desafio com cepas heterólogas virulentas a campo, que provocou doença clínica e morte nos animais do grupo controle. O desafio a campo pelo Anaplasma marginale não se mostrou muito virulento ou patogênico, não sendo possível concluir que a imunização com $\boldsymbol{A}$. centrale proteja os animais contra anaplasmose.

Palavras-chave: tristeza parasitária bovina, babesiose anaplasmose, vacina, imunização.

\section{SUMMARY}

Attenuated strains of Babesia bovis and B. bigemina and a strain of Anaplasma centrale were evaluated as immunogens against tick-borne disease. The immunization process was shown to be inocuous, immunogenic and efficient to protect cattle, at least against the babesias. Vaccinated animals proved to be protected when naturally challenged with heterologous and virulent strains, in contrast to control animals that showed clinical symptoms of the disease and had to be treated. The Anaplasma marginale challenge was not efficient since treatment was not necessary in the control animals, therefore it was not possible to conclude that immunization with A. centrale protected animals against anaplasmosis.

Key words: tick-borne disease, babesiosis, anaplasmosis, vaccine, immunisation.

\section{INTRODUÇÃO}

Segundo estudos de produção e sanidade no Brasil, Argentina e Uruguai, os produtores apontam o carrapato Boophilus microplus e os agentes por eles transmitidos, Babesia bovis, $\boldsymbol{B}$. bigemina e Anaplasma marginale, causadores do complexo Tristeza Parasitária Bovina (TPB), como um dos maiores problemas sanitários dos rebanhos (MADRUGA $\boldsymbol{e}$ t al., 1986, SPÄTH \& MANGOLD, 1986, SOLARI et al., 1992).

No Brasil, regiões e/ou microrregiões como o Rio Grande do Sul (BRASIL et al., 1982), Minas Gerais (PATARROYO et al., 1984), Mato Grosso do Sul (MADRUGA et al., 1983), Paraná (VIDOTTO $\boldsymbol{e t}$ al., 1995) e São Paulo (BARCI $\boldsymbol{e t}$ al., 1995) apresentam riscos de surto de TPB. Essa situação, em princípio, não ocorre em outras como Rio de Janeiro (VIANNA et al., 1992), Centro Oeste do Brasil (LINHARES et al., 1992), Bahia (ARAÚJO et al., 1995) e Santa Catarina (DALAGNOL et al., 1995).

Entretanto, a necessidade de aumento e melhoria da produtividade agropecuária vem causando transformações radicais no manejo dos campos (introdução de grandes áreas de agricultura e de pastagens cultivadas) e dos animais (cruzamentos industriais, com introdução de raças européias onde só havia zebuínos e vice-versa, com aumento da densidade populacional de bovinos). Estas transformações alteram de maneira importante a estabilidade em relação à sanidade do rebanho, agravando o equilíbrio parcial que existe entre bovinos e carrapatos, podendo trazer conseqüências desastrosas em relação a TPB (FURLONG \& EVANS, 1991).

\footnotetext{
${ }^{1}$ Médico Veterinário Doutor, Embrapa - Centro de Pesquisa de Pecuária dos Campos Sulbrasileiros (CPPSul). CP 242, 96400-970, Bagé, RS, Brasil. E-mail: anasacco@cppsul.embrapa.br. Autor para correspondência.

${ }^{2}$ Médico Veterinário Doutor, Embrapa - Centro Nacional de Pesquisa de Gado de Corte (CNPGC), Campo Grande, MS, Brasil Recebido para publicação em 13.09.00. Aprovado em 24.01.01
} 
Este quadro demonstra a necessidade de um método seguro de prevenção e controle da TPB, o que tem sido obtido através da utilização de cepas atenuadas de $\boldsymbol{B}$. bovis e $\boldsymbol{B}$. bigemina, obtidas segundo técnicas descritas respectivamente por CALLOW \& MELLORS (1966) e DALGLIESH $\boldsymbol{e} t$ al. (1981), e de A. centrale como imunógenos (DALGLIESH $\boldsymbol{e} \boldsymbol{t} \boldsymbol{a l} ., 1990$ e WRIGHT, 1991). No Brasil, o desenvolvimento dessas cepas atenuadas foi apresentado por KESSLER $\boldsymbol{e t}$ al. (1991 e 1998). O presente estudo apresenta os resultados da utilização desses imunógenos em animais da raça Hereford que foram desafiados naturalmente, através do carrapato, no campo.

\section{MATERIAL E MÉTODOS}

O experimento foi realizado no Centro de Pesquisa de Pecuária dos Campos Sulbrasileiros (CPPSul/EMBRAPA), em Bagé, RS, no período de fins de primavera, verão/outono. Foram utilizadas novilhas da raça Hereford, com aproximadamente 24 meses de idade, adquiridas em propriedade livre de B. microplus, e sorologicamente negativas aos agentes da TPB.

Para a imunização contra a babesiose, foram utilizadas as cepas CG-7271 (B. bovis, 17 passagem) e CG-7257 (B. bigemina, 6 ${ }^{\text {a }}$ passagem), cujos processos de isolamento e atenuação estão descritos em KESSLER et al. (1987 a e b). Para a imunização contra a anaplasmose, foi utilizada uma cepa de A. centrale, lote 7285, cedida pelo DILAVE-Miguel C. Rubino, Uruguai. Todas estas cepas foram conservadas em nitrogênio líquido adicionando-se como crioprotetores $8 \%$ de polivinilpirrolidona (PVP) e $12 \%$ de glicerol. O inóculo de imunização constou de $1 \times 10^{7}$ eritrócitos parasitados por cada um dos agentes: $\boldsymbol{B}$. bovis e $\boldsymbol{B}$. bigemina atenuadas e $\boldsymbol{A}$. centrale. Foi descongelado em banho-maria, a $37^{\circ} \mathrm{C}$ e aplicado, por via subcutânea, no mesmo período do descongelamento.

Delineamento experimental: Vinte novilhas foram aleatoriamente distribuídas para formar dois grupos de 10 animais. O grupo 1 foi inoculado com B. bovis + B. bigemina atenuadas + A. centrale. O grupo 2 não foi inoculado (grupo testemunha). Os dois grupos foram mantidos juntos, desde a chegada no CPPSUL até 93 dias após a imunização, em campo livre de carrapatos e, como medida profilática, banhados por imersão, com produto à base de amitraz, a cada 10 dias. Quando do desafio, todos foram transferidos para outro campo, naturalmente infestado com larvas de $\boldsymbol{B}$. microplus, não recebendo mais banhos carrapaticidas. Como as espécies do gênero
Anaplasma têm ciclo mais prolongado que as do gênero Babesia, as reações de imunização e desafio foram analisadas em duas fases: na fase 1, considerada do dia zero ao $40^{\circ}$ dia após a imunização e desafio, estão os resultados decorrentes da reação à Babesia spp. Na fase 2, considerada do dia 41 a 93 após a imunização e 41 a 173 após o desafio, estão os resultados decorrentes da reação ao Anaplasma spp. A avaliação do processo foi realizada através do monitoramento dos parâmetros: temperatura retal, volume globular e nível de parasitemia, determinados três vezes por semana (diariamente, quando necessário, na reação ao desafio), determinação de anticorpos específicos no soro (semanal) e peso corporal (mensal). Os resultados foram analisados por análise de variância $(\mathrm{P}<0,05)$.

\section{RESULTADOS}

\section{Processo de imunização}

Não houve diferença significativa entre as médias de temperatura retal nos dois grupos em nenhuma das fases, porém os animais do grupo 2 apresentaram médias de temperatura significativamente maiores que as iniciais (Tabela 1).

Tabela 1 - Média das variações de temperatura retal $(\mathrm{T})$ e peso corporal $(\mathrm{kg})$, porcentagem de queda no volume globular (VG), porcentagem de parasitemia (PP) e período prépatente médio (PPP), soroconversão (\%) e títulos medianos (IFI - Imunofluorescência indireta) dos animais dos grupos 1 (imunizado com $\boldsymbol{B}$. bovis $+\boldsymbol{B}$. bigemina atenuadas $+A$. centrale) e 2 (não imunizado testemunha) nas fases 1 (de reação às babesias) e 2 (de reação aos anaplasmas) durante o período de imunização.

\begin{tabular}{lccccc}
\hline & & \multicolumn{2}{c}{ Fase 1} & \multicolumn{2}{c}{ Fase 2 } \\
\cline { 3 - 6 } Parâmetros & G1 & G2 & G1 & G2 \\
& & Imunizado & Controle & Imunizado & Controle \\
\hline T & & 0,5 & 0,3 & 0,5 & 0,4 \\
VG & & $-21^{\mathrm{B}}$ & $-6^{\mathrm{A}}$ & $-34^{\mathrm{B}}$ & $-12^{\mathrm{A}}$ \\
PP & Bo & $<0,1$ & - & - & - \\
PPP & Bo & 14,3 & - & - & - \\
PP & Bi & 0,3 & - & - & - \\
PPP & Bi & 11,4 & - & - & - \\
PP & Ac & - & - & 3,6 & $<0,1$ \\
PPP & Ac & - & - & 35,3 & 65,3 \\
IFI & Bo & $100(1: 1280)$ & - & - & - \\
IFI & Bi & $70(1: 80)$ & - & - & - \\
IFI & Am & - & - & $100(1: 320)$ & - \\
Peso & & 11,0 & 12,0 & 24,0 & 29,0 \\
\hline
\end{tabular}

Letras desiguais indicam diferenças significativas $(\mathrm{P}<0,05)$ entre os grupos, dentre cada fase.

$\mathrm{Bo}=\boldsymbol{B}$. bovis $\mathrm{Bi}=\boldsymbol{B}$. bigemina $\mathrm{Am}=A$. marginale $\mathrm{Ac}=A$. centrale 
O volume globular dos animais do grupo 1 , nas fases 1 e 2 , apresentou queda significativa, entretanto os valores normais foram recuperados sem auxílio de tratamento. Estes valores mínimos coincidiram com os períodos de maior parasitemia e temperaturas retais máximas, ou logo após estes. Os animais do grupo 2 não apresentaram queda nos valores de $\mathrm{VG}$ na fase 1 , mas sim na fase 2 , coincidindo com um período pós parasitemia por $\boldsymbol{A}$. centrale, pois embora estes animais não tenham sido inoculados experimentalmente, apresentaram parasitemia (Tabela 1).

No grupo 1, houve parasitemia patente por B. bovis, B. bigemina e $\boldsymbol{A}$. centrale em 30, 50 e $100 \%$ dos animais, respectivamente. Neste período, na fase 1 , nenhum animal do grupo 2 apresentou parasitemia por nenhum dos três agentes. Na fase 2 , $70 \%$ dos animais do grupo 2 apresentaram parasitemia por A. centrale. Estas parasitemias foram detectadas 28 dias após as primeiras parasitemias no grupo inoculado (Tabela 1).

Em relação à resposta humoral, houve produção de anticorpos (IgG) anti- B. bovis, B. bigemina e $A$. marginale nos animais do grupo 1, sendo que os animais do grupo 2 permaneceram negativos neste período. Os títulos de anticorpos anti-B. bovis foram altos (até 1:5120) e constantes em $100 \%$ dos animais. Os anticorpos anti-A. marginale se apresentaram constantes, porém não tão elevados quanto os anti-B. bovis. Quanto a resposta a B. bigemina, apesar de títulos de 1:1280 terem sido observados, a resposta padrão esteve em torno de 1:40 e 1:80 em $60 \%$ dos animais; $40 \%$ apresentaram reações de títulos muito baixos e inconstantes. No encerramento da fase de imunização, somente $70 \%$ dos animais apresentaram reação sorológica positiva para $\boldsymbol{B}$. bigemina (Tabela 1).

Os animais dos dois grupos apresentaram ganho de peso, sem diferença significativa entre eles (Tabela 1).

\section{Processo de desafio}

$\mathrm{Na}$ fase 1, a análise de variância diária entre os dois grupos demonstrou que, nos dias 28, 29, 31 e 35 pós desafio, coincidindo com a parasitemia máxima em $60 \%$ do grupo, os animais do grupo 2 apresentaram temperatura retal significativamente maior que os do grupo 1 . Os animais dos dois grupos apresentaram aumento cada fase. significativo de temperaturas em relação às suas temperaturas iniciais, porém, no grupo 2 , os animais mantiveram-se com temperaturas elevadas por um período de tempo significativamente maior $(5,2$ dias vs 2,6 dias). Na fase 2, os animais tiveram aumento significativo de temperatura, sem diferença entre os grupos (Tabela 2).

$\mathrm{Na}$ fase 1, os dois grupos apresentaram queda significativa nos valores de VG em relação a seus valores iniciais, porém nos animais do grupo 2 , esta queda não só foi significativamente maior, como se manteve por um período de tempo significativamente maior que no grupo 1 (15 dias vs 10 dias). Na fase 2, os dois grupos apresentaram queda significativa nos valores de VG, sem diferença entre eles (Tabela 2).

Houve parasitemia patente por B. bovis, B. bigemina e A. marginale em 70, 100 e 100\% dos animais, respectivamente, no grupo 1 . No grupo 2, $100 \%$ dos animais apresentaram parasitemia pelos três agentes (Tabela 2).

$\mathrm{Na}$ resposta humoral, os animais do grupo 1 mantiveram e aumentaram a positividade das reações apresentadas na imunização e os animais do grupo 2 começaram a apresentar as primeiras reações positivas, sendo que a resposta ao antígeno de $\boldsymbol{B}$. bigemina continuou sendo a mais fraca e inconstante (Tabela 2).

Tabela 2 - Média das variações de temperatura retal $(\mathrm{T})$ e peso corporal $(\mathrm{kg})$, porcentagem de queda no volume globular (VG), porcentagem de parasitemia (PP) e período pré-patente médio (PPP), soroconversão (\%) e títulos medianos (IFI - Imunofluorescência indireta) dos animais dos grupos 1 (imunizado com $\boldsymbol{B}$. bovis $+\boldsymbol{B}$. bigemina atenuadas $+\boldsymbol{A}$. centrale) e 2 (não imunizado - testemunha) nas fases 1 (de reação às babesias) e 2 (de reação aos anaplasmas) durante o período de desafio.

\begin{tabular}{|c|c|c|c|c|c|}
\hline & & \multicolumn{2}{|c|}{ Fase 1} & \multicolumn{2}{|l|}{ Fase 2} \\
\hline \multicolumn{2}{|c|}{ Parâmetros } & $\begin{array}{c}\text { G1 } \\
\text { Imunizado }\end{array}$ & $\begin{array}{c}\text { G2 } \\
\text { Controle }\end{array}$ & $\begin{array}{c}\text { G1 } \\
\text { Imunizado }\end{array}$ & $\begin{array}{c}\mathrm{G} 2 \\
\text { Controle }\end{array}$ \\
\hline \multicolumn{2}{|c|}{$\mathrm{T}$} & $2,1^{\mathrm{B}}$ & $2,4^{\mathrm{A}}$ & 0,8 & 1,0 \\
\hline \multicolumn{2}{|c|}{ VG } & $-39^{\mathrm{A}}$ & $-64^{\mathrm{B}}$ & -18 & -29 \\
\hline $\mathrm{PP}$ & Bo & 0,2 & 0,4 & - & - \\
\hline PPP & Bo & 25,4 & 21,4 & - & - \\
\hline PP & $\mathrm{Bi}$ & 0,7 & 0,4 & - & - \\
\hline PPP & $\mathrm{Bi}$ & 23,0 & 22,0 & - & - \\
\hline $\mathrm{PP}$ & Am & - & - & 1,9 & 6,7 \\
\hline PPP & $\mathrm{Am}$ & - & - & 63,7 & 67,7 \\
\hline IFI & Bo & 100(1:1280) & $100(1: 2560)$ & - & - \\
\hline IFI & $\mathrm{Bi}$ & $100(1: 320-1: 640)$ & $100(1: 160)$ & - & - \\
\hline IFI & Am & - & - & $100(1: 640-1: 1280)$ & $100(1: 1280)$ \\
\hline \multicolumn{2}{|c|}{ Peso } & $-3,0^{\mathrm{A}}$ & $-18,0^{\mathrm{B}}$ & $-2,0$ & $-3,0$ \\
\hline
\end{tabular}

Letras desiguais indicam diferenças significativas $(\mathrm{P}<0,05)$ entre os grupos, dentre

$\mathrm{Bo}=\boldsymbol{B}$. bovis $\mathrm{Bi}=\boldsymbol{B}$. bigemina $\mathrm{Am}=\boldsymbol{A}$. marginale $\mathrm{Ac}=\boldsymbol{A}$. centrale 
$\mathrm{Na}$ fase 1, houve perda de peso nos animais dos dois grupos, entretanto, no grupo 2, a perda de peso foi significativamente maior. Na fase 2, os animais dos dois grupos também apresentaram perda de peso, porém sem diferença significativa entre eles (Tabela 2).

O processo de imunização não provocou doença clínica em nenhum dos animais inoculados, que recuperaram seus valores normais de VG naturalmente, sem utilização de medicamentos. O processo de desafio provocou babesiose clínica em $90 \%$ dos animais do grupo 2, com necessidade de tratamento específico e auxiliar (soroterapia e protetores hepáticos), sendo que, destes, um morreu. No grupo 1, os animais recuperaram seus parâmetros normais sem necessidade de nenhum tratamento.

$\mathrm{Na}$ fase 2, os animais apresentaram diferenças significativas de temperatura retal, de valores de VG e de peso em relação aos valores iniciais, mas sem diferença entre os grupos. Nenhum animal, nos dois grupos, apresentou necessidade de tratamento específico para anaplasmose.

\section{DISCUSSÃO}

Quando da reação ao inóculo das babesias, os animais não apresentaram alterações significativas de temperatura, o que também foi descrito por De VOS et al. (1982), TIMMS et al. (1983), JORGENSEN et al. (1989), PAYNE $\boldsymbol{e t}$ al. (1990) e MANGOLD et al. (1992). Já ao contrário, AGUIRRE et al. (1991) descreveram aumento moderado de temperatura retal nos animais imunizados. A queda significativa nos valores de volume globular, de $21 \%$ em média, foi a alteração mais marcante do processo de imunização e está de acordo com outros valores descritos, como de $18,7 \%$ e 21,3\% (GUGLIELMONE et al., 1989), 25\%; (PAYNE $\boldsymbol{e} \boldsymbol{t} \boldsymbol{a l} ., 1990)$ e 21,75\% (AGUIRRE $\boldsymbol{e} \boldsymbol{t} \boldsymbol{a l}$., 1991).

A infectividade das amostras atenuadas de Babesia utilizadas não pôde ser comprovada pela detecção de parasitemia patente, pois somente $30 \%$ dos animais apresentaram-se positivos para B. bovis e $50 \%$ para B. bigemina, ao contrário do que descreveram GUGLIELMONE et al. (1989), MANGOLD et al. (1990) e AGUIRRE $\boldsymbol{e t}$ al. (1991), segundo os quais $100 \%$ dos animais apresentaram parasitemia por B. bovis e 79\%, $83 \%$ e $92 \%$, respectivamente, apresentaram-se positivos para $\boldsymbol{B}$. bigemina. Mesmo os períodos pré-patentes médios aqui descritos são diferentes, mais longos que os apresentados nos trabalhos de GUGLIELMONE $\boldsymbol{e}$ t al. (1989). Segundo os estudos desses autores, os períodos foram de 10,3 dias em $\boldsymbol{B}$. bovis e 7,8 dias em B. bigemina, e as parasitemias foram maiores, de $1,4 \%$ e $1,5 \%$. Também MANGOLD et al. (1990) apresentaram períodos mais curtos, de 9,6 a 11,8 dias em B. bovis e 5 dias em B. bigemina. É possível que as amostras utilizadas neste experimento, que induziram parasitemias menores com períodos pré-patentes maiores, sejam mais virulentas que as utilizadas pelos autores acima, pois RODRIGUEZ $\boldsymbol{e t} \boldsymbol{a l}$. (1983) e BUENING $\boldsymbol{e t} \boldsymbol{a l}$. (1986) demonstraram uma correlação entre menor virulência e diminuição de prepatência e RISTIC \& MONTENEGRO-JAMES (1988) descrevem que amostras com maior número de passagens pelo processo de atenuação apresentam um maior nível ou parasitemia no sangue periférico.

De VOS et al. (1982), trabalhando com $\boldsymbol{B}$. bigemina atenuada, correlacionaram períodos prépatentes maiores e mais variados com amostras de baixa infectividade. De fato, pode-se considerar a amostra de B. bigemina utilizada neste trabalho como sendo de baixa infectividade e imunogenicidade, pois não foi capaz de induzir de maneira constante a formação de anticorpos específicos, os quais apresentaram títulos com valores medianos fracos de 1:80 em apenas $70 \%$ dos animais. Entretanto, a amostra de $\boldsymbol{B}$. bovis atenuada, apesar de ter demonstrado parasitemia patente baixa e em apenas $30 \%$ dos animais, mostrou alta imunogenicidade, induzindo a formação constante de anticorpos específicos de títulos com valores medianos de 1:1280 em 100\% dos animais ao final da imunização, demonstrando que todos os animais foram infectados. A infectividade é muito importante nos imunógenos atenuados de Babesia spp, sendo que CALLOW \& TAMMEMAGI (1967) enfatizam a relação entre infecção e subseqüente imunidade dos animais.

O processo de imunização, apesar das manifestações clínicas discretas ou não, como no caso do volume globular, não alterou o ganho de peso dos animais, e estes apresentaram recuperação plena de todos os parâmetros analisados sem necessidade de tratamento específico, o que também foi descrito por GUGLIELMONE et al. (1989), ABDALA (1989), AGUIRRE et al. (1991) e SOLARI et al. (1992). Ao contrário, no trabalho descrito por PAYNE et al. (1990), foi necessário o tratamento específico de $18 \%$ dos animais no período de reação às babesias, sendo a maioria dos tratamentos em resposta a infecção patente por $\boldsymbol{B}$. bovis.

Quando da reação ao inóculo de $\boldsymbol{A}$. centrale, os animais não apresentaram alterações significativas de temperatura retal, mas sim queda significativa nos valores de volume globular, resultado semelhante aos encontrados por PIPANO et al. (1985) e ABDALA et al. (1990). A 
infectividade da amostra de A. centrale foi comprovada pela detecção de parasitemia patente e presença de anticorpos específicos em 100\% dos animais. O período pré-patente médio de 35,3 dias é comparável ao observado por GLOSS (1964), que trabalhou com sangue fresco de animais doadores, por PIPANO et al. (1985), que trabalharam com sangue resfriado por 24 horas e por ABDALA et al. (1990), que trabalharam com sangue fresco e congelado. Por outro lado, a parasitemia média de $3,6 \%$ só se assemelha à descrita por ABDALA et al. (1990), sendo que os outros autores encontraram parasitemias bem maiores ou menores, demonstrando uma grande variação neste parâmetro.

Deve ser ressaltado que os animais do grupo testemunha, contaminados por $\boldsymbol{A}$. centrale, no período de imunização, apresentaram aumento de temperatura retal e queda nos valores de volume globular, em relação aos parâmetros iniciais. A queda nos valores de volume globular poderia ter sido causada pela parasitemia indevida, ainda que esta tenha sido baixa, pois PIPANO et al. (1985) observaram que $58,5 \%$ dos animais inoculados com A. centrale tiveram parasitemias abaixo de $1 \%$, como a apresentada aqui, e queda média nos valores de volume globular de 26,08\%. Entretanto, esta parasitemia indevida não explicaria o aumento de temperatura retal, pois os animais do grupo inoculado apresentaram parasitemias médias maiores sem aumento significativo de temperatura retal. Embora AGUIRRE et al. (1991) não tenham constatado a transmissão de $\boldsymbol{A}$ centrale entre animais inoculados e não inoculados, mantidos juntos por quatro meses nos períodos de primavera / verão, época de alta incidência de dípteros hematófagos, o mais provável é que a contaminação aqui descrita tenha acontecido através de insetos hematófagos, pois durante o período de imunização não houve contaminação por Babesia spp e os animais permaneceram sem carrapatos.

O desafio, através da inoculação de amostra virulenta pela exposição direta ao carrapato, a campo, foi eficiente em infectar e provocar, nos animais dos dois grupos, quadro clínico de babesiose. No grupo controle, $90 \%$ dos animais reagiram ao inóculo de desafio com manifestações clínicas severas. Na fase 1 , os animais do grupo controle apresentaram aumento de temperatura retal de $2,4^{\circ} \mathrm{C}$, que se manteve, em média, por 5,2 dias, o que é considerado febre moderadamente severa (KELLY, 1974). Os valores de volume globular apresentaram uma queda média de $64 \%$, sendo que os animais permaneceram, em média por 15 dias, com valores de volume globular $46 \%$ abaixo do normal. Estas manifestações clínicas não foram ainda mais severas porque os animais receberam medicação específica e, ainda assim, houve uma morte. Como consequiência deste quadro, os animais apresentaram perda de peso corporal neste período.

O desafio, que foi bastante virulento, conforme demonstrado pelas reações do grupo controle, também afetou os animais do grupo imunizado, que apresentaram febre moderadamente severa e queda significativa nos valores de volume globular. Entretanto, a imunização prévia destes animais permitiu que eles pudessem controlar a temperatura retal elevada (que se manteve por um período de tempo significativamente menor neste grupo) e a queda dos valores de volume globular (que foi significativamente menor e por um período de tempo também significativamente menor que no grupo controle). Como conseqüência destas manifestações, também estes animais apresentaram perda de peso corporal neste período, porém, significativamente menor do que a ocorrida no grupo controle. Embora estas manifestações clínicas, menos severas que no grupo controle, também tenham se manifestado em $90 \%$ dos animais do grupo imunizado, estes se recuperaram sem necessidade de tratamento, ao contrário do que aconteceu aos animais do grupo controle.

Estes resultados indicam que o processo de imunização, mesmo não tendo impedido a reação ao desafio em $90 \%$ dos animais, foi eficiente em protegê-los contra babesiose e que estes imunógenos atenuados podem ser utilizados com segurança e eficiência na imunização de bovinos. Estas conclusões também foram apresentadas por NARI $\boldsymbol{e} t$ al. (1979), MELLORS et al. (1982), JORGENSEN et al. (1989), PAYNE et al. (1990), KESSLER et al. (1991) e VANZINI et al. (1993). Porém, ficou demonstrado que amostras de $\boldsymbol{B}$. bigemina atenuadas podem apresentar falhas de infectividade, não levando à imunização dos animais, o que também é descrito por MANGOLD et al. (1990), GUGLIELMONE et al. (1991) e AGUIRRE $\boldsymbol{e t}$ al. (1991).

Ao desafio por $\boldsymbol{A}$. marginale, todos os animais, inclusive os testemunhas, controlaram a infecção sem auxílio de tratamento específico. Considera-se ainda que os animais, nos dois grupos, já vinham debilitados da fase anterior, em decorrência da babesiose. Como, em geral, o $\boldsymbol{A}$. marginale é uma rickettsia muito patogênica, que se manifesta com anemia profunda e morte dos animais sensíveis, estes resultados poderiam ser atribuídos ao fato de os animais do grupo inoculado terem sido imunizados e $70 \%$ dos animais testemunhas terem sido indevidamente inoculados pelo $\boldsymbol{A}$. centrale no período de imunização, o que os teria imunizado 
também, ainda que esta parasitemia indevida não tenha induzido à formação de anticorpos específicos detectáveis. É possível também que, no desafio, os animais tenham se infectado com amostra de baixa virulência de A. marginale. Assim, não foi conclusivo que a imunização com $\boldsymbol{A}$. centrale tenha protegido os animais do desafio por $\boldsymbol{A}$. marginale, o que foi demonstrado no trabalho de ABDALA et al. (1990).

\section{CONCLUSÃO}

A imunização de bovinos com amostras atenuadas de $\boldsymbol{B}$. bovis e $\boldsymbol{B}$. bigemina mais $\boldsymbol{A}$. centrale, preservadas com glicerol, em nitrogênio líquido, demonstrou ser inócua, imunogênica e eficiente, pelo menos no que diz respeito às babesias. Ainda que a amostra de $\boldsymbol{B}$. bigemina atenuada tenha sido considerada de baixa infectividade e imunogenicidade, é possível que tenha havido infecção e subseqüente imunidade, pois ao desafio todos os animais do grupo vacinado apresentaram parasitemias de até $0,7 \%$ por $\boldsymbol{B}$. bigemina sem requerer tratamento específico para recuperação, como o que aconteceu no grupo testemunha.

\section{REFERÊNCIAS BIBLIOGRÁFICAS}

ABDALA, A.A. Evolución del peso corporal y circunferencia escrotal en bovinos machos vacunados y no vacunados contra babesiosis y anaplasmosis. Veterinaria Argentina, v.6, n. 58, p.527-531, 1989

ABDAlA, A.A., PIPANO, E., AGUIRRE, D.H. et al. Frozen and fresh Anaplasma centrale vaccines in the protection of cattle against Anaplasma marginale infection. Revue Élev Med Vet Pays trop, v.43, n.2, p.155-158, 1990.

AGUIRRE, D.H., MANGOLD, A.J., RIOS, L.G. de $\boldsymbol{e t}$ al. Respuesta clínica y evolución del peso corporal en terneras (Bos taurus) vacunadas simultaneamente contra babesiosis y anaplasmosis con inmunógenos vivos. Medicina Veterinária, v.8, n.2, p.95-101, 1991.

ARAÚJO, F.R., MADRUGA, C.R., MIGUITA, M. et al. Prevalência de anticorpos contra Babesia bovis e Babesia bigemina em bovinos no Estado da Bahia. Revista Brasileira de Parasitologia Veterinária, v.4, n.2, suplemento 1, p.187, 1995.

BARCI, L.A.G., DELL'PORTO, A., FUJII, T.U. et al. Epidemiologia da babesiose bovina no Estado de São Paulo: estudo em rebanhos de corte da região do Vale do Ribeira. Revista Brasileira de Parasitologia Veterinária, v.4, n.2, suplemento 1, p.205, 1995.

BRASIL, A.G., MONMANY, L.F., SÁ, M.L.G. et al. Premunição contra a tristeza parasitária em bovinos a campo. A Hora Veterinária, ano 2, n.10, p.4-8, 1982.
BUENING, G.M., KUTTLER, K.L., RODRIGUEZ, S.D. Evaluation of a cloned Babesia bovis organism as a live immunogen. Veterinary Parasitology, v.22, p.242, 1986.

CALLOW, L.L., MELLORS, L.T. A new vaccine for Babesia argentina infection prepared in splenectomised calves. Australian Veterinary Journal, v.42, p.464-465, 1966.

CALLOW, L.L., TAMMEMAGI, L. Vaccination against bovine babesiosis. Infectivity and virulence of blood from animals either recovered from or reacting to Babesia argentina. Australian Veterinary Journal, v.43, p.249-256, 1967.

DALAGNOL, C.A., MARTINS, E., MADRUGA, C.R Prevalência de anticorpos contra Babesia bovis, Babesia bigemina, Anaplasma marginale em bovinos de corte na região de clima C \& b. Revista Brasileira de Parasitologia Veterinária, v.4, n.2, suplemento 1, p.220, 1995.

DALGLIESH, R.J., CALLOW, L.L., MELLORS, L.T. et al. Development of a highly infective Babesia bigemina vaccine of reduced virulence. Australian Veterinary Journal, v.57, n.1, p.8-11, 1981.

DALGLIESH, R.J., JORGENSEN, W.K., De VOS, A.J Australian frozen vaccines for the control of babesiosis and anaplasmosis in cattle - a review. Tropical Animal Health Production, v.22, p.44-52, 1990.

De VOS, A.J., COMBRINK, M.P., BESSENGER, R. Babesia bigemina vaccine: comparison of the efficacy and safety of Australian and South Africa strains under experimental conditions in South Africa. Onderstepoort Journal of Veterinary Research, v.49, p.155-158, 1982.

FURLONG, J., EVANS, D. Epidemiologia do carrapato Boophilus microplus no Brasil: necessidade de uma abordagem compreensível para seu estudo realístico. In. SEMINÁRIO BRASILEIRO DE PARASITOLOGIA VETERINÁRIA, 7, SIMPÓSIO SOBRE A MOSCA-DOSCHIFRES Haematobia irritans, 2, 1991, São Paulo, Brasil. Anais... São Paulo : Colégio Brasileiro de Parasitologia Veterinária, 1991. p.48-50.

GLOSS, R.M. Nota prévia - Experiências com o Anaplasma centrale com finalidade de facilitar o serviço de premunição. In. CONFERÊNCIA DA SOCIEDADE VETERINÁRIA DO RIO GRANDE DO SUL-SOVERGS, 3, 1964, Porto Alegre, RS. Anais... Porto Alegre : SOVERGS, 1964. p.66-71.

GUGLIELMONE, A.A., MANGOLD, A.J., AGUIRRE, D.H. $\boldsymbol{e}$ al. Vacunación simultánea con Babesia bovis y Babesia bigemina atenuadas, congeladas en nitrógeno líquido con DMSO o glicerol como crioprotectores. Revista de Medicina Veterinaria, v.70, n.4, p.176-182, 1989.

GUGLIELMONE, A.A., MANGOLD, A.J., AGUIRRE, D.H. et al. Vacunas congeladas contra la babesiosis y la anaplasmosis de los bovinos: viabilidad luego de la descongelación. Revista Cubana de Ciencias Veterinarias, v.22, n.3, p.233-240, 1991.

JORGENSEN, W.K., De VOS, A.J., DALGLIESH, R.J. Infectivity of cryopreserved Babesia bovis, Babesia bigemina and Anaplasma centrale for cattle after thawing, dilution and incubation at $30^{\circ} \mathrm{C}$. Veterinary Parasitology, v.31, n.3-4, p.243-251, 1989. 
KELLY, W.R. Veterinary clinical diagnosis. Second edition. Baillière Tindall, London. 1974. Cap.4, p.21-26.

KESSLER, R.H., MADRUGA, C.R., De JESUS, E.F. $\boldsymbol{e}$ t al. Isolamento de cepas puras de Babesia bovis, Babesia bigemina e Anaplasma marginale em área enzoótica. Pesquisa Agropecuária Brasileira, Brasília, v.22, n.7, p.747-752, 1987a.

KESSLER, R.H., SACCO, A.M.S., De JESUS, E.F. $\boldsymbol{e}$ t al. Desenvolvimento de cepas vivas atenuadas de Babesia bovis e Babesia bigemina: teste preliminar. Pesquisa Agropecuária Brasileira, Brasília, v.22, n.11-12, p.1225$1230,1987 \mathrm{~b}$

KESSLER, R.E., SASTRE, A.M., MOREIRA, M.A. $\boldsymbol{e} \boldsymbol{t} \boldsymbol{a l}$. Experiencias con vacunas vivas atenuadas de Babesia bovis, Babesia bigemina, Anaplasma marginale y Anaplasma centrale conservadas por congelación en Brasil. Revista Cubana de Ciencias Veterinárias, v.22, n.3, p.189-196, 1991.

KESSLER, R.H., SACCO, A.M.S., MADRUGA, C.R., $\boldsymbol{e t}$ al Teste crítico de vacinas atenuadas de Babesia bovis, B. bigemina e Anaplasma marginale em novilhas da raça Holandesa. Revista Brasileira de Parasitologia Veterinária, v.7, n.1, p.1-5, 1998.

LINHARES, G.F.C., MASSARD, C.L., ARAÚJO, J.L. de B. et al. Levantamento sorológico para Babesia bigemina (Smith \& Kilborne, 1893) e Babesia bovis (Babés, 1888) em bovinos na Região Centro-Oeste do Brasil. Arquivos da Universidade Federal Rural do Rio de Janeiro, v.15, n.1, p.85-91, 1992.

MADRUGA, C.R., AYCARDI, E., PUTT, N. Epidemiologia da anaplasmose e babesiose em bovinos da Região de Cerrados do Estado de Mato Grosso do Sul : I - Prevalência Arquivos Brasileiros de Medicina Veterinária e Zootecnia , v.35, n.5, p.631-640, 1983.

MADRUGA, C.R., BERNE, M.E.A., KESSLER, R.H. $\boldsymbol{e}$ t $\boldsymbol{a l}$. Diagnóstico da tristeza parasitária bovina no Estado de Mato Grosso do Sul: inquérito de opinião. Fundação Cargill, 1986. 40p. il. (EMBRAPA-CNPGC. Circular Técnica, n.18).

MANGOLD, A.J., AGUIRRE, D.H., GUGLIELMONE, A.A. Post-thawing viability of vaccines for bovine babesiosis and anaplasmosis cryopreserved with glycerol. Veterinary Parasitology, v.37, p.301-306, 1990.

MANGOLD, A.J., BERMÚDEZ, A.C., ANZIANI, O.S. $\boldsymbol{e} \boldsymbol{t}$ al. Efectos clínicos de Babesia bigemina vacunal o patógena en novillos Holando Argentino. Revista de Medicina Veterinária, v.73, n.1, p.43-45, 1992.

MELLORS, L.T., DALGLIESH, R.J. TIMMS, P. et al Preparation and laboratory testing of a frozen vaccine containing Babesia bovis, Babesia bigemina and Anaplasma centrale. Research in Veterinary Science, v.32, n.2, p.194197, 1982.

NARI, A. SOLARI, M.A., CARDOZO, H. Hemovacuna para el control de Babesia spp y Anaplasma marginale en el Uruguay. Veterinaria (Montevideo), v.15, p.137-148, 1979.
PATARROYO, J.H., SANTOS, J.L., RIBEIRO, M.F.B. et al. Diagnóstico da situação sanitária bovina do Estado de Minas Gerais. VI - Aspectos epidemiológicos da babesiose bovina na "Zona da Mata". In: CONGRESSO BRASILEIRO DE MEDICINA VETERINÁRIA, 19, 1984, Belém, Pará. Resumos... Belém-Pará : Faculdade de Ciências Agrárias do Pará, Julho 1984. p.226.

PAYNE, R.C., OSORIO, O., YBAÑEZ, A. Tick-borne diseases of cattle in Paraguay. II. Immunization against anaplasmosis and babesiosis. Tropical Animal Health and Production, v.22, p.101-108, 1990.

PIPANO, E., MAYER, E., FRANK, M Comparative response of Friesian milking cows and calves to Anaplasma centrale vaccine. The British Veterinary Journal, v.141, p.174-178, 1985.

RISTIC, M., MONTENEGRO-JAMES, S. Immunization against Babesia. In. Babesiosis of domestic animals and man. Boca Ratón, Florida : CRC, 1988. Capítulo 10, p.163-189.

RODRIGUEZ, S.D., BUENING, G.M., GREEN, T.J. et al. Cloning of Babesia bovis by in vitro cultivation. Infection and Immunity, v.42, p.15, 1983.

SOLARI, M.A., NARI, A., CARDOZO, H. Impact of Babesia bovis and Babesia bigemina on the production of beef cattle in Uruguay. Memórias do Instituto Oswaldo Cruz, Rio de Janeiro, v.87, supplement III, p.143-149, 1992.

SPÄTH, E.J.A., MANGOLD, A.J. Algunas caracteristicas de producción y sanidad de rodeos de ganado bovino de carne en el Sudeste de Salta y norte de Tucumán. Revista Argentina de Producción Animal, v.6, n.1-2, p.101-112, 1986.

TIMMS, P., DALGLIESH, R.J., BARRY, D.N. et al. Babesia bovis: comparison of culture-derived parasites, non-living antigen and conventional vaccine in the protection of cattle against heterologous challenge. Australian Veterinary Journal, v.60, p.75-77, 1983.

VANZINI, V.R., RAMÍREZ, L.M., DRAGHI DE BENITEZ, M.G. Poder inmunógeno de dos cepas atenuadas en Babesia bovis en bovinos Hereford. Revista de Medicina Veterinária, v.74, n.2, p.58-62, 1993.

VIANNA, L.F.C.G., FACCINI, J.H.L., LOPES, C.W.G. et al. Estado de premunição e estabilidade enzoótica para os hemoparasitos em bovinos mestiços de zebu na Baixada Fluminense, Estado do Rio de Janeiro. Arquivos da Universidade Federal Rural do Rio de Janeiro, v.15, n.2, p.155-165, 1992.

VIDOTTO, O., YAMAMURA, G.M.A., BARBOSA, C.da S. et al. Ocorrência de Babesia bigemina, B. bovis e Anaplasma marginale em rebanhos de bovinos leiteiros da região de Londrina, PR. Revista Brasileira de Parasitologia Veterinária, v.4, n.2, suplemento 1, p.184, 1995.

WRIGHT, I.G. Towards a synthetic Babesia vaccine. International Journal for Parasitology, v.21, n.2, p.155$159,1991$. 